\title{
Obtaining a Neural Network Mathematical Model That Takes Into Account Various Power Supply Schemes
}

\author{
G.A. Kilin", B.V. Kavalerov, A.I. Suslov, and M.A. Kolpakova \\ Perm National Research Polytechnic University, 614990 Perm, Russia
}

\begin{abstract}
Gas turbine units are widely used as a drive for a synchronous generator in a gas turbine power plant. The main problem here lies in the fact that the control systems of such gas turbine plants are transferred practically unchanged from their aviation counterparts. This situation leads to inefficient operation of the gas turbine power plant, which affects the quality of electricity generation. To solve this problem, it is necessary to improve the control algorithms for the automatic control systems of gas turbine plants. When solving this problem, gas turbine plants should be considered in interaction with other subsystems and units; for gas turbine power plants, this is, first of all, an electric generator and the electric power system as a whole. Setting up a control system is one of the most costly stages of their production, both in terms of finance and time. Especially time-consuming operations are non-automated manual configuration management system for developmental and operational testing. Therefore, it is proposed to use a software-modeling complex, on the basis of which it is possible to obtain a neural network mathematical model of a gas turbine power plant and conduct its tests.
\end{abstract}

\section{Introduction}

The design and development of the electric power industry requires the introduction of modern control algorithms for gas turbine units (GTU) as part of gas turbine power plants (GTPP). For the synthesis of such algorithms, it is necessary to carry out iterative procedures for testing the GTPP as a control object [1-4]. In the presence of a mathematical model (MM), the procedure for setting up and testing the control system is greatly simplified, since the direct operation of a real object is excluded. But there are high requirements for this MM. On the one hand, it should reproduce all the typical operating modes of a gas turbine power plant, and on the other hand, it should be fast-acting for its direct use in GTU's automatic control system's (ACS) control circuit. Using the theory of neural networks [5 - 9] allows to obtain such mathematical models $[10,11]$. In addition, neural networks make it possible to diagnose GTU as part of GTES [12, 13].

\footnotetext{
${ }^{*}$ Corresponding author: thisisforasm@rambler.ru
} 
It should be noted that previously obtained models were always adequate only for a specific power supply scheme and a specific operating mode [5 - 7]. Subsequently, a model was obtained that takes into account several modes of operation of the power system [12], on the basis of which conclusions were drawn about the possibility of obtaining an all-mode neural network model of the power system. The analysis of the results obtained led to the assumption of the possibility of obtaining such a neural network model that takes into account various power supply schemes.

\section{Methods}

An artificial neural network (ANN), which is used to obtain a neural network model of a GTPP (0) as part of a software modeling complex (SMC) (0), allows you to flexibly customize its architecture to achieve the required adequacy and learning rate.

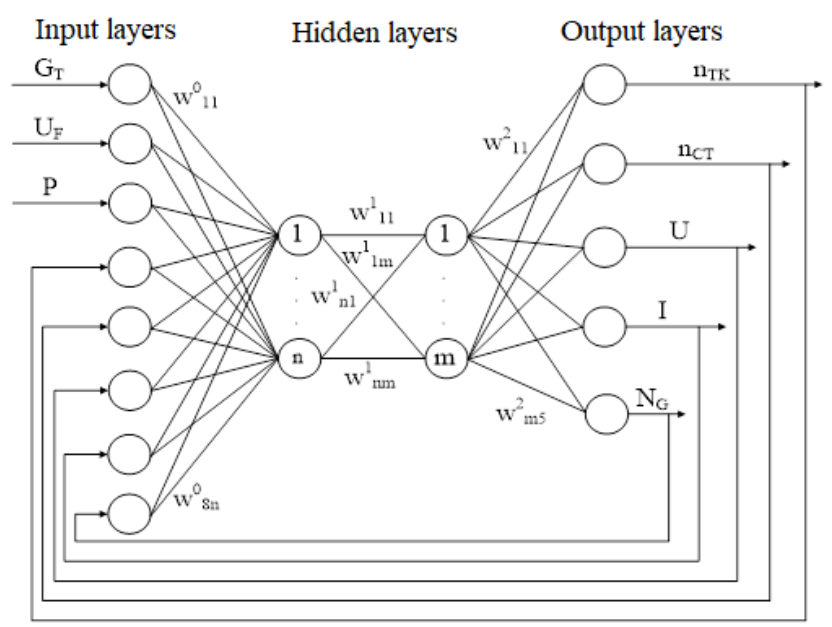

Fig. 1. Neural network architecture for modeling a gas turbine power plant

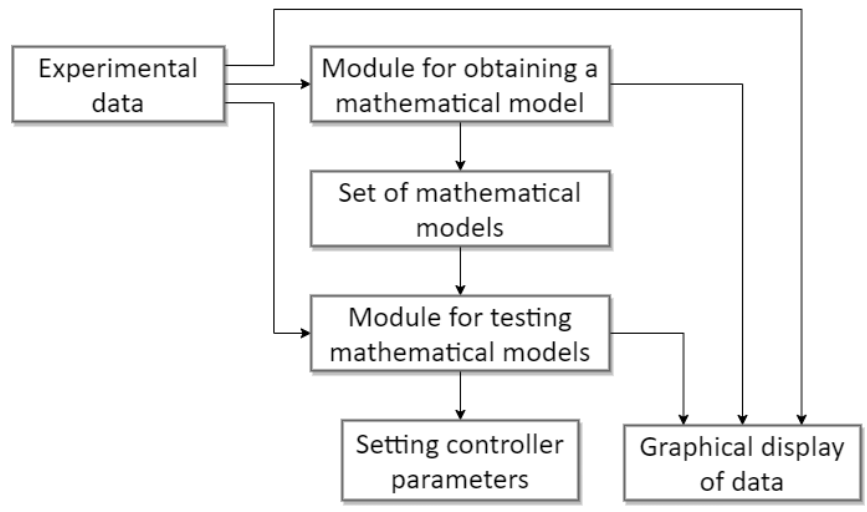

Fig. 2. Functional diagram of the SMP modules 
To justify the choice of the ANN architecture, a large number of experiments were carried out $[5-7,12]$ and, taking into account the recommendations $[8,9]$, the following conclusions were obtained:

1. Use at least two hidden layers;

2. Mandatory presence of feedbacks;

3. The depth of feedbacks must be at least six;

4. The number of neurons in the hidden layer can vary over a wide range, but 30 neurons are enough to solve our problem.

\section{Results and Discussion}

Two power supply schemes with different modes were considered as a source of experimental data:

1. GTPP operates on a dedicated load, power dropping mode of active-inductive load from $6000 \mathrm{~kW}$ to $1000 \mathrm{~kW}$;

2. GTPP operates on a dedicated load in parallel with a infinite power network, power surge mode of active-inductive load from $1000 \mathrm{~kW}$ to $2000 \mathrm{~kW}$;

In article [12], in which a multi-mode model of a GTPP and a power system was obtained, the architecture of a neural network with 2 hidden layers and 30 neurons in each hidden layer was used. Therefore, the same architecture was applied to solve the current problem. Unfortunately, with the current architecture, it was not possible to obtain a model of acceptable adequacy [13]. To obtain a neural network mathematical model of the GTPP and the power system, several experiments were carried out with a different number of hidden layers and neurons in these layers:

1. Architecture 2: 2 hidden layer, 40 neurons

2. Architecture 3: 3 hidden layer, 30 neurons

3. Architecture 4: 3 hidden layer, 40 neurons

4. Architecture 5: 4 hidden layer, 30 neurons

5. Architecture 6: 4 hidden layer, 40 neurons

After the experiments, the following conclusions were made:

1. Despite the increased number of neurons, the power of architecture 1 was not enough to obtain a model of acceptable adequacy [13];

2. On average, an increase in the number of neurons in each layer improves the adequacy of the model;

3. An increase in the number of neurons in each layer increases the serial number of the resulting model with better adequacy in the experiment.

The results of experiment No. 2 showed acceptable adequacy according to Theil's criterion [13] (Table 1) and this model is suitable for testing its performance in a closed loop.

Table. 1. Adequacy according to the Theil's criterion

\begin{tabular}{|c|c|c|}
\hline \multicolumn{2}{|c|}{ Variable } & Adequacy measure \\
\hline Free turbine rotation speeds & Training sample & 0,03212 \\
\cline { 2 - 3 } & Test sample & 0,02125 \\
\hline \multirow{2}{*}{$\begin{array}{c}\text { Line voltage of synchronous } \\
\text { generator }\end{array}$} & Training sample & 0,02389 \\
\cline { 2 - 3 } & Test sample & 0,02176 \\
\hline
\end{tabular}


Figures 3 - 6 show a comparison of experimental and model data on training and test data, all experiments were carried out with the GTU's ACS turned off.

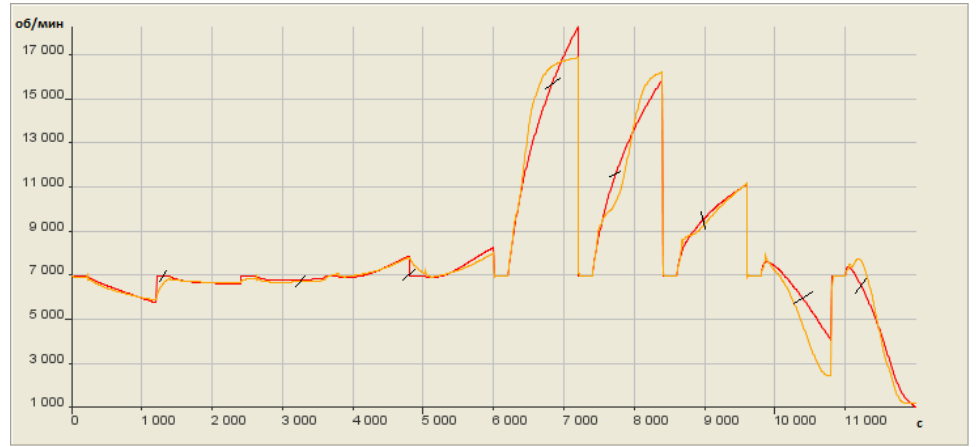

Fig. 3. Change in the rotation speed of a free turbine in the training set for modes: load surge 10002000, load shedding 6000-1000.

Experimental data -

Model data -

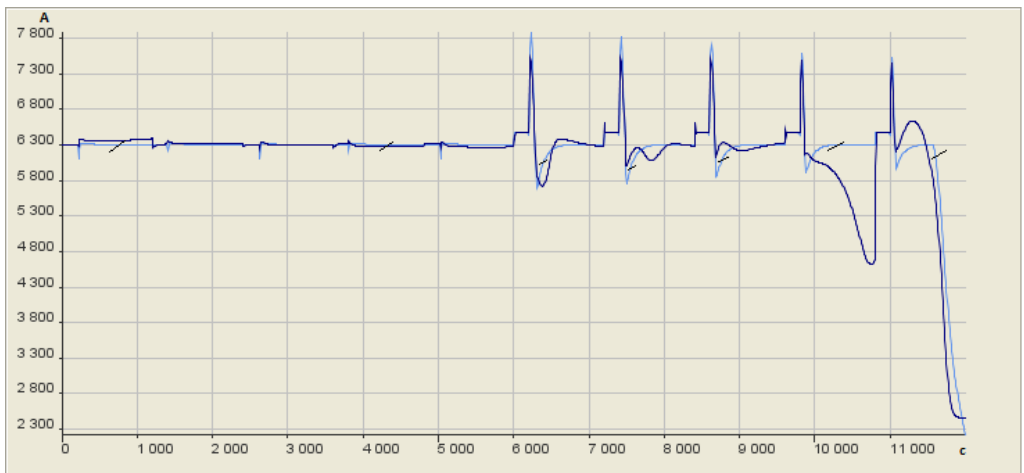

Fig. 4. Change in voltage of the synchronous generator in the training sample for modes: load surge 1000-2000, load shedding 6000-1000.

Experimental data -

Model data -
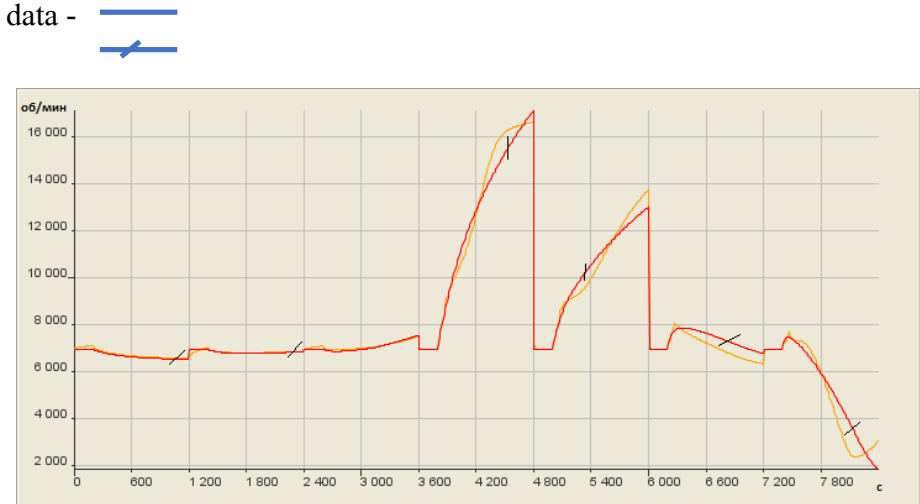

Fig. 5. Changing the rotation speed of a free turbine in the test sample for modes: load surge 10002000 , load shedding 6000-1000. 
Experimental data -

Model data -

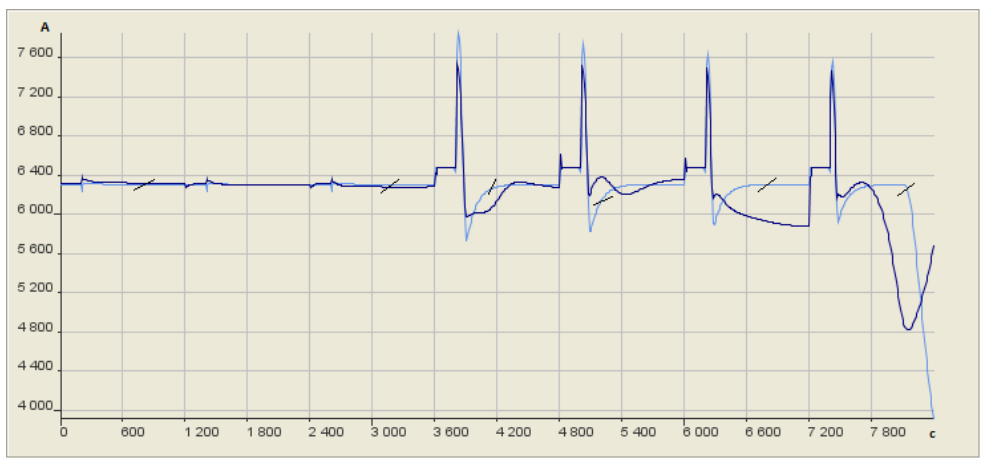

Fig. 6. Change of voltage of the synchronous generator in the test sample for modes: load surge 10002000, load shedding 6000-1000.

Experimental data

Model data -

Figures 7 - 10 show a comparison of experimental and model data in a closed loop with the ACS turned on.

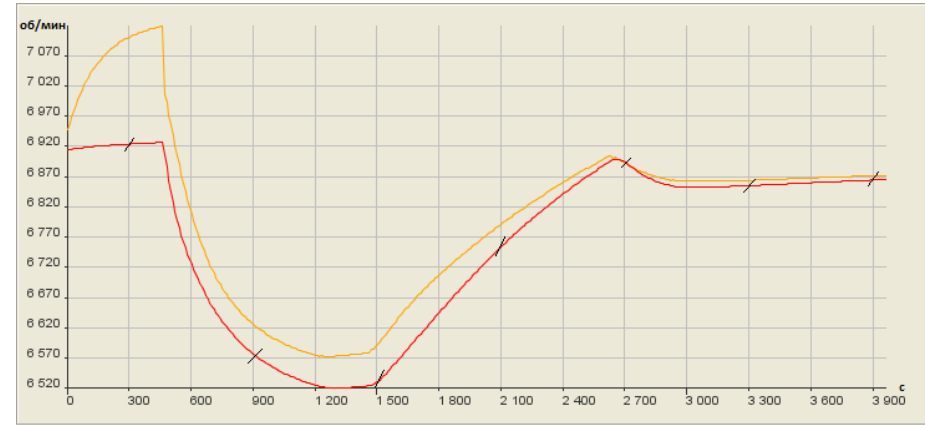

Fig. 7. Changing the rotation speed of a free turbine in a closed loop with an operating ACS for a load surge mode 1000-2000.

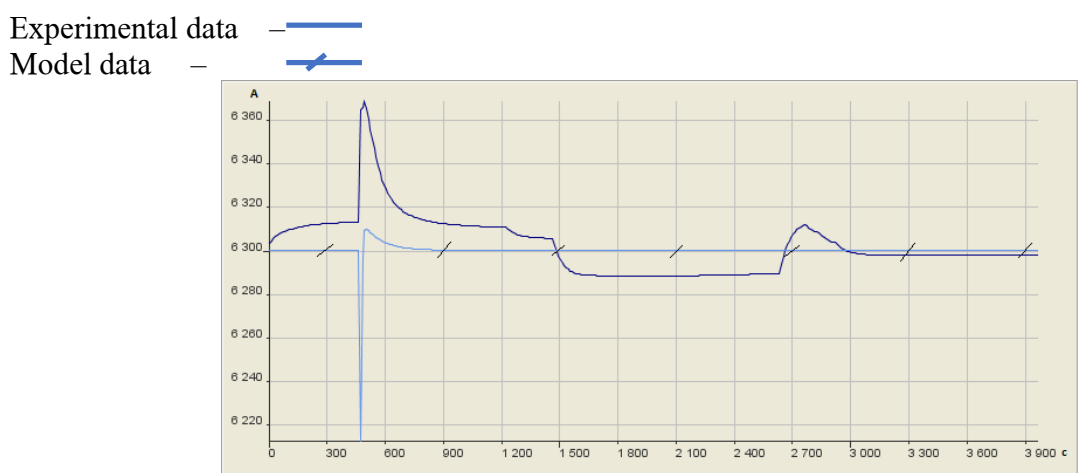

Fig. 8. Change in voltage of a free turbine in a closed loop with an operating ACS for a load surge mode 1000-2000. 
Experimental data $-\overline{-}$

Model data

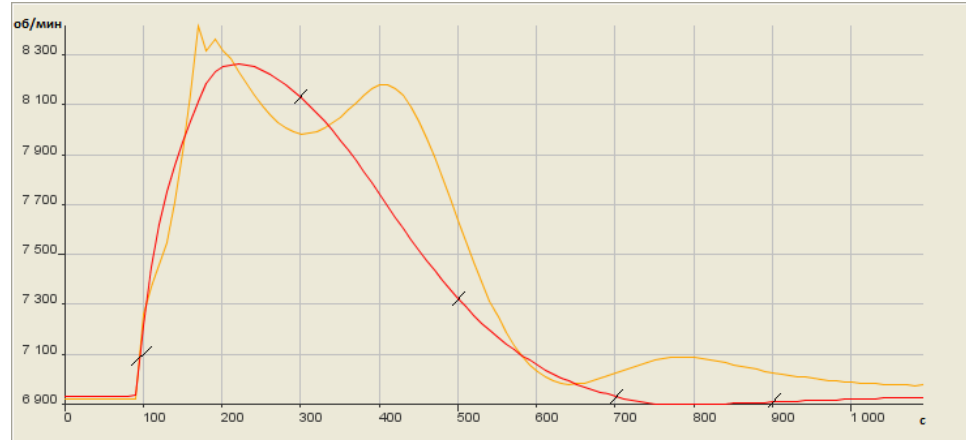

Fig. 9. Changing the rotation speed of a free turbine in a closed loop with an operating ACS for load shedding mode 6000-1000.

Experimental data $-\square$
Model data -

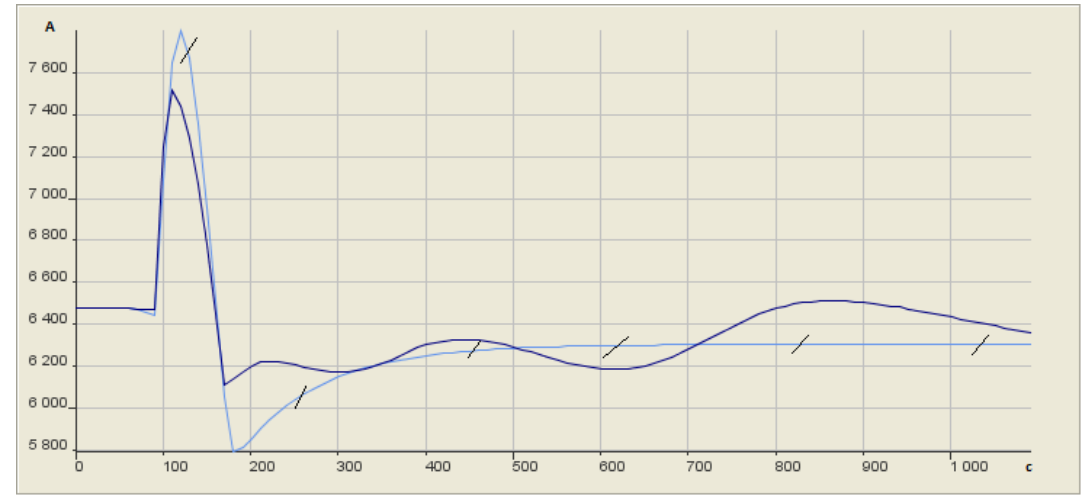

Fig. 10. Change in voltage of a free turbine in a closed loop with an operating ACS for load shedding mode 6000-1000.

Experimental data

Model data

\section{Conclusions}

The results obtained allow us to assume that within the framework of one neural network, we can simulate not only most modes of operation of a power system, but also various schemes of such a power system. In the long term, this gives us a huge advantage, since it allows us to obtain a universal neural network mathematical model of a power system, which should significantly simplify the testing procedure for such a power system. The main difficulty in obtaining such a model lies in the required amount of experimental data that must be obtained somewhere, as well as in the training time, since the amount of experimental data will be significant. 


\section{Acknowledgements}

The study was carried out with the financial support of the Russian Foundation for Basic Research and the Perm Territory within the framework of the scientific project № 19-48-590012.

\section{References}

1. B. V. Kavalerov, A. V. Romodin, Bulletin of the North-Eastern Federal University, 8(3), 42 (2011)

2. D. V. Voskoboinikov, Fundamental research: Limited Liability Company Publishing House "Academy of Natural Science", 2, 3926 (2015)

3. A. I. Polulyakh, I. G. Lisovin, B. V. Kavalerov, A.A. Shigapov, Bulletin of the Voronezh State Technical University Voronezh: Voronezh State Technical University, 7,11.1, 129 (2011)

4. D. I. Volkov, V. M. Grudinkin, V. A. Kachura, A. A. Razladsky, Aerospace engineering and technology Kharkiv, 9, 133 (2008)

5. G. A. Kilin, I. V. Bakhirev, B. V. Kavalerov, Automation in electric power and electrical engineering: Perm National Research Polytechnic University, 1, 72 (2015)

6. G. A. Kilin, B. V. Kavalerov, K. A. Odin, Bulletin of the Perm National Research Polytechnic University, Electrical engineering, information technology, control systems: Perm National Research Polytechnic University, 2, 7 (2014)

7. G. A. Kilin, Bulletin of Kalashnikov ISTU: Federal State Budgetary Educational Institution of Higher Professional Education "Izhevsk State Technical University named after M.T. Kalashnikov ", 2, 87 (2015)

8. S. Haykin, Neural networks: a complete course, 2 (2008)

9. V. V. Kruglov, V. V. Borisov, Artificial neural networks. Theory and practice (2001)

10. H. Asgari, X.Q. Chen, M. B. Menhaj, R. Sainudiin, Journal of Engineering for Gas Turbines and Power: American Society of Mechanical Engineers, 135(9), 092601 (2013)

11. H. Asgari, X.Q. Chen, R. Sainudiin, International Journal of Modeling, Identification and Control: Inderscience, 20(3), 253 (2013)

12. M. B. Rahmoune, A. Hafaifa, M. Guemana, Advances in Acoustics and Vibration. Springer, Cham, 1 (2017)

13. F. Zhao, Gas turbine exhaust system health management based on recurrent neural networks, Procedia CIRP, 83, 630 (2019)

14. S. Kiakojoori, K. Khorasani, Neural Computing and Applications, 27(8), 2157 (2016)

15. E. A. Zaborovtsev, M. A. Kolpakova, G. A. Kilin, Bulletin of the Perm National Research Polytechnic University, Electrical engineering, information technology, control systems, 34, 217 (2020)

16. G. Tail, Economic forecasts and decision making (1971) 